\section{Take Control Over Your Fluconazole Prescriptions: The Growing Importance of Candida glabrata as an Agent of Candidemia in Brazil}

To the Editor-Several studies have been performed to document the epidemiology of candidemia in Brazil..$^{1-3}$ Investigations performed in different regions of the country yielded similar results: Candida albicans is the most common cause of candidemia, responsible for roughly half of the cases, followed by Candida parapsilosis and Candida tropicalis. The proportion of cases of candidemia caused by Candida glabrata has typically been less than $5 \%$. In contrast, C. glabrata is the second or third leading cause of candidemia in many developed countries. ${ }^{4}$ The lower consumption of fluconazole in the developing world might at least partially explain these differences. Since fluconazole use has increased by an average of $21 \%$ per year in our institution, we aimed to determine if any secular change occurred in the incidence of candidemia due to C. glabrata.

Santa Casa Complexo Hospitalar is a 1,200-bed tertiary care university hospital located in Southern Brazil. It is composed of 7 hospitals dedicated to distinct medical specialties. The institution assists mostly patients admitted through the public health care system (approximately $60 \%$ of all patients), but also insured and private patients.

This study was divided into 2 periods. The first period included all Candida bloodstream infections diagnosed from January 1, 1995, through December 31, 2003. These data have already been published. ${ }^{1,5,6}$ The second period included all cases of candidemia diagnosed from July 1, 2005, through December 31,2007. Patients were included only once in the study, and no significant change in laboratory methods occurred during the study.

Candidemia was detected by using the BacT/ALERT blood culture system, and species identification was performed with ID $32 \mathrm{C}$ (both bioMérieux). To investigate any potential association between the rate of fluconazole consumption and the incidence of candidemia due to C. glabrata, cases were grouped according to the hospital where the infection occurred. The defined daily dose of fluconazole was set at $400 \mathrm{mg}$, and the denominator was 1,000 patient-days. Only data for inpatients were available. Since fluconazole dosage varies largely on the basis of body weight in children, pediatric patients were not studied. In addition, it should be noted that $C$. glabrata candidemia is very unusual in children.

During the first period of study (1995-2003), C. glabrata was the etiologic agent of $8(3.5 \%)$ of 228 cases of candidemia. However, this proportion was 3-fold greater during 2005-2007; it accounted for $28(10.6 \%)$ of 263 cases of candidemia. These cases were not equally distributed among study hospitals. As shown in the Figure, the highest incidence of candidemia due to C. glabrata infection occurred in the transplant hospital, followed by the lung hospital and the cancer hospital. The incidence of candidemia due to Candida krusei remained low during the study.

The Figure shows that, after adjustment for defined daily dose per 1,000 patient-days, hospitals with a relatively higher rate of consumption of fluconazole also exhibited a greater incidence of candidemia due to $C$. glabrata. These variables were strongly correlated (Pearson correlation coefficient, $0.905 ; P=.01$ ).

A significant increase in fluconazole minimum inhibitory concentrations has been observed in C. glabrata isolates in recent years by means of the broth dilution antifungal susceptibility testing method approved by the Clinical and Laboratory Standards Institute ${ }^{7}$. The median minimum inhibitory concentration of fluconazole in 2005 was $4 \mu \mathrm{g} / \mathrm{mL}$ (interquartile range [IQR], $4-8 \mu \mathrm{g} / \mathrm{mL}$ ); in 2006 , it was $4 \mu \mathrm{g} / \mathrm{mL}$ (IQR, $4-8 \mu \mathrm{g} / \mathrm{mL}$ ); and in 2007 , it was $8 \mu \mathrm{g} / \mathrm{mL}$ (IQR, $8-8 \mu \mathrm{g} / \mathrm{mL}$ ) $(P=.03$, Kruskal-Wallis test).

As far as we know, this is the first study to document the emergence of $C$. glabrata as an important cause of fungemia in Latin America. There was a statistically significant concentra-

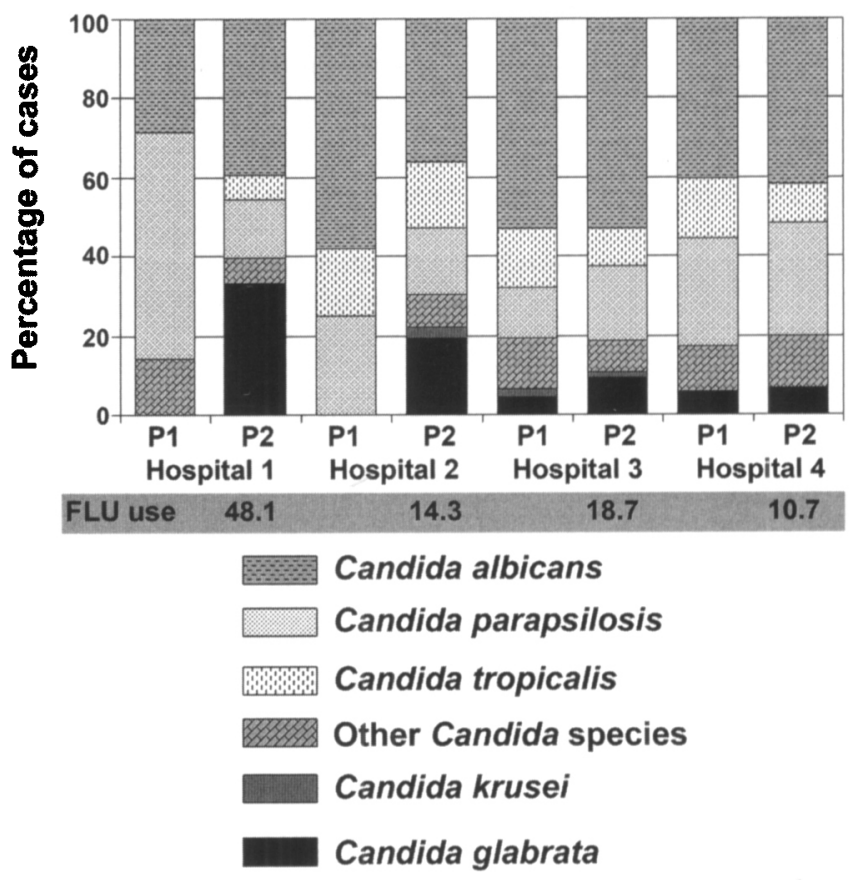

FIGURE. Distribution of Candida species causing candidemia in 4 of the main study hospitals (transplant, 1; lung, 2; cancer, 3; and general, 4) during the 2 study periods of 1995-2003 (P1) and 2005-2007 (P2). Rates of fluconazole (FLU) use are stated as defined daily doses per 1,000 patient-days. The correlation between the rate of FLU use and the incidence of $C$. glabrata candidemia was statistically significant $(P=.03)$. 
tion of cases in those wards experiencing a heavy use of fluconazole. Despite the well-accepted theoretical association between fluconazole overuse and changes in the epidemiology of candidemia, a surprisingly low number of studies have addressed this issue, and the results have been somewhat conflicting. For instance, Sendid et al. ${ }^{8}$ demonstrated that increasing use of prophylaxis with fluconazole was associated with a reduction in candidemia prevalence. This was related to an increase in the number of cases of C. glabrata candidemia. Although the authors provided the defined daily dose of fluconazole, ${ }^{8}$ there was no adjustment in terms of patient-days. Conversely, other studies have failed to demonstrate any association between fluconazole use and the emergence of $C . g l a-$ brata candidemia. ${ }^{9,10}$

Since $C$. glabrata candidemia may emerge in centers where fluconazole is intensively used, should not infection control professionals audit the use of this antifungal agent as seriously as they audit the use of many antibacterial drugs? We believe this could most benefit centers where the incidence of $C$. glabrata infection remains low. There are several clinical situations where the use of fluconazole could be restricted. One example is the treatment of candiduria: except for very specific situations, antifungal drugs are generally useless in this context. Another situation is oral candidosis, a condition in which exposure to topical agents plays an important role. Fluconazole prophylaxis should also be avoided in low-risk situations. Furthermore, it is essential that hospital staff adhere to the institutional protocols for the rational use of antifungal drugs.

Our study has several limitations, the first of which is its retrospective design. Although the reasons for a higher incidence of candidemia in recent years are not clear, inadequate care of central venous catheters may play an important role. Actually, our center has one of the highest incidences of candidemia in Brazil (unpublished data), and the incidence of catheter-related bacterial bloodstream infections is also quite high. Unfortunately, no data about outpatient use of fluconazole were available. Other variables not studied, such as underlying diseases and severity of illness, might also have influenced the distribution of species causing candidemia. Also, the increase in cases due to C. glabrata could be simply a consequence of an outbreak occurring in some of our hospitals. Despite these limitations, there was a clear association between increased fluconazole use and an increased incidence of candidemia due to $C$. glabrata. A prospective trial evaluating strategies for rational use of fluconazole and the subsequent impact of fluconazole consumption (measured in defined daily doses per 1,000 patient-days) on the distribution of species causing candidemia would be of inestimable value in clarifying these issues.

\section{ACKNOWLEDGMENTS}

Potential conflicts of interest. In the last 5 years, Dr. Pasqualotto has been paid for speaking on behalf of Pfizer, United Medical (Gilead), and Merck, and he has received research grants from Pfizer, Fungal Research Trust, and SigmaTau. All other authors report no conflicts of interest relevant to this article.

Alessandro C. Pasqualotto, $\mathrm{MD}, \mathrm{PhD}$; Ricardo A. Zimerman, MD; Sydney H. Alves, PharmD, PhD; Valerio R. Aquino, MSc; Daniela Branco, RN; Denusa Wiltgen, MD, MSc; Angelica do Amaral, RN; Raquel Cechinel, RN; Soraya M. Colares, MD; Ivana G. da Rocha, BSc; Luiz Carlos Severo, MD, PhD; Teresa Cristina T. Sukiennik, MD

From the Infection Control department (A.C.P., R.A.Z., D.B., D.W., A.doA., R.C., S.M.C., I.G.daR., T.C.T.S. ) and the Mycology Laboratory (L.C.S.), Santa Casa Complexo Hospitalar, and the Universidade Federal do Rio Grande do Sul (A.C.P., L.C.S.), the Conselho Nacional de Desenvolvimento Científico e Tecnologico (A.C.P., L.C.S.), and the Mycology Laboratory, Hospital de Clínicas de Porto Alegre (V.R.A.), Porto Alegre; and the Mycology Laboratory, Universidade Federal de Santa Maria (S.H.A.), Santa Maria; Rio Grande do Sul, Brazil.

Address reprint requests to Alessandro C. Pasqualotto, $\mathrm{MD}, \mathrm{PhD}$, Serviço de Controle de Infecção Hospitalar (SCIH), Santa Casa Complexo Hospitalar, $7^{\circ}$ andar, Porto Alegre, 90035-075, Brazil (pasqualotto@santacasa.tche.br).

Infect Control Hosp Epidemiol 2008; 29:898-899

(c) 2008 by The Society for Healthcare Epidemiology of America. All rights reserved. 0899-823X/2008/2909-0018\$15.00. DOI: 10.1086/590191

\section{REF E RENCES}

1. Pasqualotto AC, de Moraes AB, Zanini RR, Severo LC. Analysis of independent risk factors for death among pediatric patients with candidemia and a central venous catheter in place. Infect Control Hosp Epidemiol 2007;28:799-804.

2. Antunes AG, Pasqualotto AC, Diaz MC, d'Azevedo PA, Severo LC. Candidemia in a Brazilian tertiary care hospital: species distribution and antifungal susceptibility patterns. Rev Inst Med Trop Sao Paulo 2004;46:239241.

3. Colombo AL, Guimarães T, Silva LR, et al. Prospective observational study of candidemia in São Paulo, Brazil: incidence rate, epidemiology, and predictors of mortality. Infect Control Hosp Epidemiol 2007;28:570576.

4. Colombo AL, Nucci M, Park BJ, et al. Epidemiology of candidemia in Brazil: a nationwide sentinel surveillance of candidemia in eleven medical centers. J Clin Microbiol 2006;44:2816-2823.

5. Pasqualotto AC, Nedel WL, Machado TS, Severo LC. A comparative study of risk factors and outcome among outpatient-acquired and nosocomial candidaemia. J Hosp Infect 2005;60:129-134.

6. Pasqualotto AC, Nedel WL, Machado TS, Severo LC. A 9-year study comparing risk factors and the outcome of paediatric and adults with nosocomial candidaemia. Mycopathologia 2005;160:111-116.

7. NCCLS. Reference method for broth dilution antifungal susceptibility testing of yeasts. Approved standard. 2nd ed. Wayne, PA: NCCLS, 2002: M27-A2.

8. Sendid B, Cotteau A, François N, et al. Candidaemia and antifungal therapy in a French University Hospital: rough trends over a decade and possible links. BMC Infect Dis 2006;6:80.

9. Blot $\mathrm{S}$, Janssens R, Claeys G, et al. Effect of fluconazole consumption on long-term trends in candidal ecology. J Antimicrob Chemother 2006;58: $474-477$.

10. Marchetti O, Bille J, Fluckiger U, et al. Epidemiology of candidemia in Swiss tertiary care hospitals: secular trends, 1991-2000. Clin Infect Dis 2004;38:311-320. 